\title{
Letter to the Editor concerning "Intraosseous basivertebral nerve ablation for the treatment of chronic low back pain: a prospective randomized double-blind sham-controlled multi-center study" by Fischgrund JS, et al. [Eur Spine J; (2018) 27(5): 1146-1156]
}

\author{
Yongchao Li ${ }^{1}$ Xiaofei Feng ${ }^{1}$ · Jun Tan ${ }^{1} \cdot$ Baogan Peng ${ }^{2}$
}

Received: 22 June 2019 / Revised: 22 June 2019 / Accepted: 2 July 2019 / Published online: 15 July 2019

(c) Springer-Verlag GmbH Germany, part of Springer Nature 2019

We read with great interest the study by Fischgrund JS et al., which appeared in the European Spine Journal. In this prospective randomized double-blind sham-controlled multicenter study, they demonstrated the efficacy of intraosseous radiofrequency ablation of the basivertebral nerve to treat chronic low back pain in patients with Modic type 1 or 2 changes of the vertebral endplates. At the same time, treatment was limited to a minimum of two and a maximum of three consecutive vertebral body levels from L3-S1. Most commonly, L5-S1 (122/224 patients) and L4-L5 (45/224) followed by L4-L5-S1 (41/224) were treated. In other words, a total of $163 \mathrm{~S} 1$ vertebral bodies $(72.77 \%)$ were treated. Targeted location was the branch point or terminus of the basivertebral nerve, typically between 40 and $60 \%$ of the posterior to anterior distance across the vertebral body.

However, the S1 vertebrae are anatomically and functionally distinct from the lumbar vertebrae. In a human anatomical study, Degmetich et al. found in 2016 that unlike the lumbar vertebral body, S1 vertebral body has no posterior nutrient foramen that facilitates basivertebral nerve penetration through the vertebral cortex. Rather, nerves penetrate the $S 1$ via the lateral aspects (the sacral foramens), consistent with being left and right branches of the anterior sacral nerve.
Although Fischgrund JS et al. provided a new way to treat chronic low back pain, overall, the effect was not very significant. In the 12 months after treatment, the ODI score of the treatment group decreased by 19.8 and that of the control group decreased by 15.9; the VAS score of the treatment group decreased by 2.76 and that of the control group decreased by 2.16 . Therefore, in order to achieve better clinical efficacy, the target position of the intraosseous radiofrequency ablation should be placed differently in the S1 and lumbar vertebral body. The neurotherapy target of S1 should be located at the mid-lateral borders of the S1 vertebral body instead of the mid-posterior border.

\section{Compliance with ethical standards}

Conflict of interest None declared.

Publisher's Note Springer Nature remains neutral with regard to jurisdictional claims in published maps and institutional affiliations.

Jun Tan

dr.tan@139.com

$\triangle$ Baogan Peng

pengbaogan76611@163.com

1 Department of Orthopaedics, Shanghai East Hospital, Tongji University School of Medicine, 150 Jimo Road, Shanghai 200120, China

2 Department of Spinal Surgery, The Third Medical Center of PLA General Hospital, 69 Yongding Road, Beijing 100039, China 